\title{
Word order variation in spatial descriptions with adverbs
}

\author{
ROBIN HÖRNIG, THOMAS WESKOTT, REINHOLD KLIEGL, and GISBERT FANSELOW \\ University of Potsdam, Potsdam, Germany
}

\begin{abstract}
Previous research has shown that in a three-term spatial reasoning task, the second premise of a German premise pair is especially easy to comprehend if (1) the prepositional object rather than the grammatical subject denotes the given entity, and if (2) the term denoting the given entity precedes the term denoting the new entity. Accordingly, the second premise is easiest to comprehend with noncanonical word order - that is, with the prepositional object in preverbal position denoting the given entity (e.g., To the right of the given object is the new subject). This finding is explained in terms of contextual licensing of noncanonical word order. Here, we discuss and tested two alternative accounts of contextual licensing, given-new and partially ordered set relations (Poset). The given-new account claims that noncanonical word order is licensed by the term denoting the given entity preceding the term denoting the new entity. On the Poset account, noncanonical word order is licensed if the preverbal constituent introduces a new entity that stands in a transitive, irreflexive, and asymmetric relation to a given entity. Comprehension times for second premises with spatial adverbs in four different word orders support both accounts of contextual licensing; Poset licensing was stronger than given-new licensing.
\end{abstract}

When people read a spatial relational statement, such as The snake is to the left of the deer, and when such a statement is continued by a second one, such as The donkey is to the right of the deer, they are capable of making a spatial inference-in the present instance, that the snake is to the left of the donkey. For a valid conclusion, the two statements that constitute the premise pair must meet two conditions. First, the premises must have exactly one term in common (the middle term) - the deer in the example just given. Second, the description as a whole must be determinate insofar as either premise must relate two adjacent entities of the layout to one another (i.e., the middle term must denote the inner entity of a linear layout). According to the mental model theory of reasoning (Johnson-Laird, 1983), people arrive at the conclusion by initially constructing a mental model of the layout described by the first premise. The second premise is then interpreted in the context of the initial mental model. The middle term in the second premise - the deer-denotes the given entity already included within the initial model, whereas the other term in the second premise (the end term) - the donkey - denotes a new entity not mentioned

This research was supported by the Deutsche Forschungsgemeinschaft (SFB 632). We thank Petra Grüttner, Juliane Schwiercz, and Olivia Engmann for their assistance in conducting the experiment. We thank Bruce Bridgeman and Peter Staudacher for helpful comments. We are indebted to Klaus Oberauer for inspiring discussions. Correspondence concerning this article should be addressed to R. Hörnig, University of Potsdam, Allgemeine Psychologie I, P.O. Box 6015 53, D-14415 Potsdam, Germany (e-mail: rhoernig@uni-potsdam.de).

Note-This article was accepted by the previous editorial team, when Colin M. MacLeod was Editor. previously. Processing the second premise requires the integration of the new entity into the initial model. This process of premise integration constitutes the core of the reasoning process (see Huttenlocher \& Higgins, 1971). Since the middle term anaphorically refers to a previously introduced entity, premise integration in spatial reasoning exemplifies an instance of the processing of anaphoric relations in general.

For ease of exposition and in order to keep apart the different levels of linguistic representation that are involved here, we will begin with some terminological clarifications for a sentence of the form The $x$ is to the left of the $y$. On the syntactic level, we differentiate between the external argument and the internal argument of the preposition to the left of. The external argument appears as the grammatical subject, the $x$, of the sentence. The internal argument appears as the noun phrase the $y$, which is part of the prepositional phrase. Below we will use the term (grammatical) subject to designate the external argument of the preposition and the term (prepositional) object to designate the internal argument of the preposition. Note that in The $x$ is to the left of the $y$, the subject precedes the verb (the copula to be). The grammatical subject in preverbal position identifies the word order as canonical, a point that we will return to below.

On the semantic level, we differentiate between the referent (i.e., the entity that is linguistically localized) and the relatum (i.e., the entity relative to which the referent is linguistically localized) of the expressed relation (see Miller \& Johnson-Laird, 1976, p. 379). The external argument of the preposition corresponds to the referent expression denoting the entity that plays the role of the referent. The internal argument of the preposition corresponds to the relatum expression denoting the entity that 
plays the role of the relatum. Therefore, for the sentences under consideration, a word order is defined as canonical if the referent expression is in preverbal position and the relatum expression is in postverbal position.

Until recently, experimental research on spatial reasoning exclusively employed premises with canonical word order (henceforth, canonical premises). This reliance on canonical premises is not without problems, because premise integration in spatial reasoning is subject to two linguistically motivated principles, relatum $=$ given and given-new (Hörnig, Oberauer, \& Weidenfeld, 2005). Relatum $=$ given is based on the assumption that spatial relational statements are used to perform acts of linguistic localization (see Miller \& Johnson-Laird, 1976). The linguistic localization expressed by a statement such as The donkey is to the right of the deer informs the reader or hearer about the place of the referent, the donkey. To this end, the relatum, the deer, is used to narrow the domain in which to search for the donkey. This, in turn, requires the interlocutors to know the location of the relatum; that is, the (location of the) relatum must be contextually given. Suggesting that spatial relational premises tell the reasoner where to place the mentioned entities into the mental model, Hörnig et al. hypothesized that, according to relatum $=$ given, the second premise in a spatial reasoning task is easier to integrate when its relatum expression denotes the given entity, as in (1A), than when its referent expression does, as in (1B).

1A. The donkey ${ }_{\text {new }}$ is to the right of the deer ${ }_{\text {given }}$.

1B. The deer $r_{\text {given }}$ is to the left of the donkey new $_{\text {. }}$.

The second principle, given-new, relies on the givennew strategy in language comprehension (Clark \& Haviland, 1977). As applied to spatial relational statements, the integration of the new information of the current sentence with the information already given in memory proceeds in three successive steps: First, one determines the part of the current sentence denoting the entity already given; then, one accesses the given entity within the initial mental model; and finally, one attaches the new entity to the given entity as conveyed by the statement. If the first two steps are carried out as soon as the expression denoting the given entity is encoded, integration of the new entity will be speeded up when the expression denoting the given entity precedes the expression denoting the new entity. Therefore, given-new predicts a processing advantage for a given-new order in the second premise, as in (1B), as opposed to a new-given order, as in (1 A).

As is evident from the opposite predictions of relatum $=$ given and given-new with respect to $(1 \mathrm{~A})$ and $(1 \mathrm{~B})$, a canonical second premise cannot cope with both principles at the same time. Whenever the postverbal relatum expression of a second premise denotes the given entity, as advised by relatum $=$ given, the preverbal referent expression denotes the new entity, and therefore, the premise has new-given order, as in (1A). Conversely, whenever the preverbal referent expression denotes the given entity, the premise has given-new order, but the postverbal relatum expression denotes the new entity, as in (1B). Hörnig et al. (2005) empirically validated the two principles of premise integration by manipulating the word order of German premise pairs. In German, which is rather flexible with respect to word order, the order of relatum expression and referent expression can be reversed, as in (2A) and (2B) (English word-by-word translations are given in parentheses).

\section{A. Rechts vom Hirsch ${ }_{\text {given }}$ ist der Esel $_{\text {new }}$. (Right of-the deer ${ }_{\text {given }}$ is the donkey ${ }_{\text {new }}$.) \\ 2B. Links vom Esel $_{\text {new }}$ ist der Hirsch given $_{\text {. }}$ (Left of-the donkey ${ }_{\text {new }}$ is the deer $r_{\text {given }}$.)}

In addition to canonical premises (German equivalents of [1A] and [1B]), Hörnig et al. (2005) studied premises such as $(2 \mathrm{~A})$ and $(2 \mathrm{~B})$ with reversed word order (henceforth, noncanonical premises). Premise integration was predicted to be easiest for (2A) with a preverbal relatum expression denoting the given entity, since such a premise complies with relatum $=$ given as well as with given-new. In contrast, a preverbal relatum expression denoting the new entity, as in (2B), should render integration most difficult, since such a premise violates both principles. Canonical premises, such as (1A) and (1B), were expected to be of intermediate difficulty, since they always violate one of the principles. Comprehension times for second premises confirmed these predictions. In the present article, we will elaborate on the given-new interpretation of this finding.

\section{Licensing Noncanonical Word Order: Given- New Versus Poset}

Although word order in German is relatively flexible, a sentence with a preverbal grammatical subject realizes the canonical word order of a German main clause, which is indicated by shorter comprehension times for isolated sentences with preverbal subjects than for sentences with, for example, preverbal direct objects (Bader, Meng, Bayer, \& Hopf, 2000; Hemforth, 1993; Schlesewsky, Fanselow, Kliegl, \& Krems, 2000; comparable results for other languages have been reported for Italian by De Vinzenci, 1991, for Dutch by Frazier, 1987, and for Finnish by Hyönä \& Hujanen, 1997). Hörnig et al. (2005) found the same preference for spatial relational statements. Lacking a preceding context, a canonical first premise was read more quickly than a noncanonical first premise. Obviously, comprehension of a second premise as a function of word order was affected by context. Despite its noncanonical word order, a noncanonical second premise was especially easy to understand when its preverbal relatum expression denoted the given entity and its postverbal referent expression denoted the new entity - that is, when it had a given-new order. However, a noncanonical second premise was especially difficult to comprehend when its preverbal relatum expression denoted the new entity and its postverbal referent expression denoted the given entity - that is, when it had a new-given order. This interpretation adds up to a given-new licensing account of noncanonical word order. A context is licensing, as opposed to nonlicensing, if it facilitates the com- 
prehension of a sentence with noncanonical word order to a larger extent than it facilitates the comprehension of a sentence with canonical word order, which need not be licensed. This is what Hörnig et al. observed. The context licenses the noncanonical word order of a second premise when that word order establishes a given-new order (the relatum expression denoting the given entity precedes the referent expression denoting the new entity), but the context is nonlicensing when the result is a new-given order (the relatum expression denoting the new entity precedes the referent expression denoting the given entity). On the given-new licensing account, whether noncanonical word order is licensed or not depends crucially only on whether the preverbal relatum expression denotes the contextually given entity or the new entity. In other words, given-new licensing should hold, irrespective of the position of the spatial relational expression - the spatial preposition. For the sentences under consideration, the spatial preposition cannot be separated from its internal argument (i.e., the relatum expression) for reasons of grammaticality. Since the preverbal position is occupied by both the spatial preposition and the relatum expression, it should be informative to determine the discourse status of the denotatum of the whole prepositional phrase by taking a closer look at its internal structure.

Taken as a whole, the prepositional phrase constitutes an expression denoting a place. In our example (2A) above, to the right of the deer ${ }_{\text {given }}$ denotes the place where the donkey is to be located. In differentiating between objects and places, we take spatial relations not to hold between objects, but rather between places that can be occupied by objects (e.g., Herskovitz, 1986, pp. 33ff). According to our conception of mental model construction, the initial model constructed on the basis of the first premise incorporates two places and two objects: In The snake is to the left of the deer, the place occupied by the snake is to the left of the place occupied by the deer. Integrating the second premise $(2 \mathrm{~A})$ then requires accessing the given place, the one occupied by the deer. Next, a new place to the right of the given place is added to the initial model, and finally the new entity, the donkey, is located at the new place. On this account, the preverbal constituent of the second premise (2A) - the prepositional phrase - denotes a new entity - a new place - which is spatially related to a given place. Given this somewhat mixed discourse status of the denotata of the preverbal constituent(s) (given relatum-new place), we may wonder whether given-new licensing adequately explains what is at stake here.

Prince (1999), for example, disputed the idea that given-new licenses noncanonical structures. On the basis of corpus analyses of topicalized structures with preverbal direct objects in English and Yiddish, she concluded that a preverbal direct object is licensed not in cases where it denotes a given entity, but rather in cases where it denotes a new entity that stands in a partially ordered set relation (Poset relation) to a given entity. Poset relations are defined as either transitive, reflexive, and antisymmetric, or transitive, irreflexive, and asymmetric. Spatial relations fit the latter criterion. They are (locally) transitive (if $x$ is to the left of $y$ and $y$ is to the left of $z$, then $x$ is to the left of $z$ ), irreflexive ( $x$ is not, e.g., to the left of itself), and asymmetric (if $x$ is to the left of $y$, then $y$ is not to the left of $x$ ). Hence, the preverbal prepositional phrase in (2A) is adequately characterized as denoting a new entity (a new place) that is Poset related to a given entity (a given place). This observation suggests a Poset licensing account of the finding of Hörnig et al. (2005): Noncanonical word order of a second premise with a preverbal relatum expression denoting a given entity is licensed (i.e., especially easy to comprehend) because in this case the preverbal constituent - the prepositional phrase as a whole - denotes a new entity that is Poset related (here, spatially related) to a given entity.

Before we proceed, we should concede that it is not obvious whether the "Poset" licensing account proposed here is a proper instance of the Poset licensing advocated by Prince (1999). With her account, Prince is targeting the discourse structuring function of topicalization. Primarily, she is dealing with set membership as a Poset relation implicit in discourse. Consider the following example from Prince (1999; see also Prince, 1998):

She had an idea for a project. She's going to use three groups of mice. One, she'll feed them mouse chow, just the regular stuff they make for mice. Another she'll feed them veggies. And the third she'll feed junk food.

The second sentence introduces a set of discourse entities, three groups of mice. The left-dislocated constituents in the third and fourth sentence as well as the topicalized constituent in the fifth sentence (the third) all denote entities that are members of the set of the three groups of mice introduced before and are therefore Poset related to it. We acknowledge that Poset licensing in our sense might be deemed a remote relative of Prince's own account. What we nevertheless find striking is that our Poset interpretation conforms to the condition that a preverbal constituent must meet in order to be contextually licensed according to Prince, and that our Poset interpretation deviates in a significant way from the given-new interpretation. We therefore stick to the term Poset licensing and ask the reader to eventually add inverted commas to it.

According to given-new licensing, the preverbal prepositional phrase of (2A) is licensed because the preverbal relatum expression, which forms part of it, denotes a given entity: the deer. According to Poset licensing, the preverbal prepositional phrase of (2A) is licensed because the preverbal prepositional phrase as a whole denotes a new entity that is Poset-related to a given entity: the new place to the right of the given place that is occupied by the deer. Either interpretation might account for the good comprehensibility of a noncanonical second premise with a preverbal relatum expression denoting a given entity in spite of its noncanonical word order. The empirical evidence at hand does not decide between these two interpretations, because whenever the licensing conditions of given-new are met, the licensing conditions of Poset are met, too, and vice versa. The two accounts, however, differ as to whether the position of the spatial preposition contributes to the licensing condition or not. As outlined above, on the 
given-new account, it is only for the sake of grammaticality that the relatum expression shares the preverbal position with the spatial preposition, which does not contribute to the licensing condition. On the Poset account, the preverbal constituent denotes the new place only if it comprises both, the relatum expression denoting the given element and the preposition conveying the spatial relational information. Hence, according to Poset, the position of the spatial preposition contributes to the licensing condition.

From a processing perspective, the observed benefit from a preverbal relatum expression denoting a given entity is explained by the early access to this entity in the initial mental model, regardless of whether the given entity in question is the relatum itself (given-new) or the place occupied by it (Poset). However, on the Poset account, there might be a further source of processing benefit. The new place can be added to the model as soon as it is known how the new place relates to the given place - that is, as soon as the information carried by the spatial preposition is available. Hence, from a processing perspective, the early encoding of the whole prepositional phrase might speed up not only the accessing of the given relatum, but also the adding of the new place. Testing this possibility requires promoting early access to the given relatum while retaining the spatial relational information until the end of the statement.

\section{Varying Word Order With Spatial Adverbs}

Since word order will now be of primary interest, we switch to the syntactic terminology. When a spatial relation is expressed by means of a preposition, the preposition must precede the prepositional object (henceforth abbreviated as object). Therefore, we cannot decide which of the two interpretations, given-new or Poset licensing, explains the results of Hörnig et al. (2005). However, in German it is possible to express a spatial relational statement by means of an adverb adjoined to a prepositional phrase, with its object being the relatum expression. The position of the adverb can be varied independently of the position of the object. In the present experiment, we used four different word orders of spatial relational statements with adverbs, examples of which are given below together with rough English translations in parentheses (abbreviations indicate the order of subject, $\mathrm{S}$; object, $\mathrm{O}$; and adverb, A):

(SOA) Der Esel ist vom Hirsch aus rechts. (The donkey is as from the deer right.)

(OSA) Vom Hirsch aus ist der Esel rechts. (As from the deer the donkey is right.)

(OAS) Vom Hirsch aus rechts ist der Esel. (As from the deer right is the donkey.)

(AOS) Rechts vom Hirsch aus ist der Esel. (Right as from the deer is the donkey.)

In the SOA condition, the grammatical subject is in preverbal position and the sentence is assumed to have canonical word order. SOA differs from its prepositional variant, The donkey is to the right of the deer, with respect to the position of the relational expression. Whereas the sentence final adverb in SOA follows the object, the preposition precedes the object. The other three conditions, OSA, OAS, and AOS, all have in common that the object preceding the verb marks the construction as noncanonical. In the OSA condition, although the object is preverbal, the adverb, as in SOA, is sentence final. OSA will be the critical instance for deciding between given-new and Poset licensing. In both the OAS and the AOS conditions, the object as well as the adverb are in preverbal position. In AOS, which most closely corresponds to the prepositional variant (2A), the adverb precedes the object, whereas in OAS, the adverb follows the object.

\section{Design and Predictions}

In order to avoid a terminological conflation, we restate relatum $=$ given in terms of anaphoric relations. If the relatum is given, we will speak of an object anaphor (the relatum expression denotes the given entity), and if the relatum is new, we will speak of a subject anaphor (the referent expression denotes the given entity). The two-level factor anaphor (subject or object) was crossed with the four-level factor word order, yielding eight experimental conditions. Word order was either canonical (SOA) or noncanonical (OSA, OAS, or AOS). The first premise P1 and the second premise $\mathrm{P} 2$ were always of the same word order.

We expected to obtain a relatum $=$ given advantage that is, a main effect of anaphor. P2s with an object anaphor (given relatum) should be read faster than P2s with a subject anaphor (new relatum). We also expected anaphor to interact with word order as a function of noncanonical word order's being licensed or not. SOA word order is assumed to be canonical, requiring no licensing context. Accordingly, the difference in P2 comprehension times between the licensing and the nonlicensing conditions should be larger for noncanonical word order than for canonical word order. Statistically, this would be confirmed by an interaction of anaphor with word order.

In the object anaphor condition, the context was supposed to be licensing whenever the object anaphor as well as the adverb were in preverbal position (AOS and OAS). ${ }^{1}$ This was predicted on both accounts, given-new licensing and Poset licensing. The two accounts disagreed with respect to OSA - that is, a P2 with preverbal object but sentence final adverb. According to given-new, noncanonical word order is licensed whenever the preverbal given object precedes the new subject, irrespective of the position of the adverb. Thus, in the object anaphor condition, OSA should be licensed as much as OAS and AOS, and hence, comprehensibility should be the same for OSA, OAS, and AOS. According to Poset, the preverbal constituent is licensed if it introduces a new entity that is Poset related to a given entity. With respect to spatial relational premises, a noncanonical word order of a second premise is licensed if the preverbal constituent denotes a new place. With OSA, the preverbal object anaphor denotes the relatum; but the preverbal constituent as a whole does not denote a new place, since it lacks the spatial relational information that specifies how the new place relates to 
the given place. On the Poset account, noncanonical OAS and AOS are licensed but noncanonical OSA is not. Poset thus predicts that the object anaphor condition facilitates comprehension of OAS (and AOS) to a larger extent than comprehension of OSA.

By computing repeated contrasts for the word order conditions SOA, OSA, OAS, and AOS, we will report on pairwise comparisons of SOA with OSA, of OSA with OAS, and of OAS with AOS. Given-new predicts a significant interaction of anaphor with word order for the comparison of SOA and OSA, whereas Poset predicts a significant interaction for the comparison of OSA and OAS. Neither account predicts an interaction for the comparison of OAS and AOS.

\section{METHOD}

\section{Participants}

Twenty-five persons ( 23 high school students and 2 university students), 19 female and 6 male, from 17 to 26 years old (mean, 18.9 ) took part in the experiment. They were paid $€ 5$ (about $\$ 6$ ) for their participation.

\section{Materials}

The 128 three-term items used by Hörnig et al. (2005) were also used in the present experiment, formulated by means of adverbs instead of prepositions. The three terms of an item named animals (e.g., snake, donkey, deer), fruits or vegetables (e.g., raspberry, apple, carrot), vehicles (e.g., van, carriage, bike), musical instruments (e.g., trumpet, piano, violin), or other common objects of everyday life (e.g., pipe, pencil, key). Half of the items described a determinate linear arrangement; the other half described a two-dimensional layout. Since the hypotheses under investigation should apply regardless of whether layouts are one- or two-dimensional, dimensionality was varied to broaden the generality of our findings.

The layout of each item was held constant, while its description was varied with respect to anaphor (subject vs. object) and word order (SOA, OSA, OAS, AOS), resulting in eight variants of each item (see Table 1 for an example). Word order was always the same for both premises of a pair; that is, structures were parallel for P1 and $\mathrm{P} 2$. For half of the items, the grammatical subject of $\mathrm{P} 1$ was as- signed as the antecedent for the anaphor of P2. For the other half of the items, the object was assigned as the antecedent. Table 1 presents an example with a subject antecedent, the deer, in P1. An example with an object antecedent would be obtained by replacing P1-for example, The deer is as from the snake right - by its converse, The snake is as from the deer left. Antecedent is not included as a factor in the analyses reported below, because it did not affect P2 comprehension times in the present experiment, just as it did not in the experiment of Hörnig et al. ${ }^{2}$

Item variants were assembled in eight different versions such that each version consisted of sixteen items for each cell of the experimental design of anaphor $\times$ word order (equally distributed across antecedent and dimensionality). The four different spatial adverbs rechts (right), links (left), oben (above), and unten (below) used in $\mathrm{P} 2 \mathrm{~s}$ were balanced across conditions.

\section{Procedure}

The procedure was identical to the one used by Hörnig et al. (2005). Participants were tested individually at a PC. They were told that they would read descriptions of layouts of three pictures each, with the pictures hanging on a wall above and below each other, beside each other, or in a combination thereof. The session began with four practice trials, followed by the 128 experimental trials, which were randomized for each participant. After the participant started a trial by pressing the space bar, P1 appeared centered on the screen. The participants were instructed to press the space bar as soon as they could imagine the layout (P1 comprehension time). Thereafter, $\mathrm{P} 1$ was replaced by $\mathrm{P} 2$. The participants were told to press the space bar as soon as they could imagine the whole layout (P2 comprehension time). After pressing the space bar again, the participants were tested by a picture verification task as follows. ${ }^{3}$

The task always involved pictures of the two entities that were not explicitly related to one another by either of the premises - that is, the nonantecedent of $\mathrm{P} 1, A$, and the new entity introduced by $\mathrm{P} 2$, $C$. One of them, $A$ or $C$, was displayed in the middle of the screen with the other one added to it after a delay of $1,000 \mathrm{msec}$. The relative position of the second entity was either valid or invalid with regard to the description. For one-dimensional layouts, the distance (30 pixels) between the pictures of the two entities was too short for a third picture of the same size $(120 \times 120$ pixels $)$ to be placed between them. The participants were explicitly told in advance that they should base their judgments on the relative positions of the pictured entities. By pressing the yes button or the no button, the

Table 1

An Example (One-Dimensional Layout With Subject Antecedent) of the Eight Variants (Anaphor $\times$ Word Order) of an Original German Item (Together With Rough English Translations)

\begin{tabular}{|c|c|c|c|}
\hline Word Order & First Premise (P1) & Second Premise (P2) & Anaphor \\
\hline \multirow[t]{2}{*}{ SOA } & $\begin{array}{l}\text { Der Hirsch ist von der Schlange aus rechts. } \\
\text { The deer is as from the snake right. }\end{array}$ & $\begin{array}{l}\text { Der Hirsch ist vom Esel aus links. } \\
\text { The deer is as from the donkey left. }\end{array}$ & Subject \\
\hline & & $\begin{array}{l}\text { Der Esel ist vom Hirsch aus rechts. } \\
\text { The donkey is as from the deer right. }\end{array}$ & Object \\
\hline \multirow[t]{2}{*}{ OSA } & $\begin{array}{l}\text { Von der Schlange aus ist der Hirsch rechts. } \\
\text { As from the snake is the deer right. }\end{array}$ & $\begin{array}{l}\text { Vom Esel aus ist der Hirsch links. } \\
\text { As from the donkey is the deer left. }\end{array}$ & Subject \\
\hline & & $\begin{array}{l}\text { Vom Hirsch aus ist der Esel rechts. } \\
\text { As from the deer is the donkey right. }\end{array}$ & Object \\
\hline \multirow[t]{2}{*}{ OAS } & $\begin{array}{l}\text { Von der Schlange aus rechts ist der Hirsch. } \\
\text { As from the snake right is the deer. }\end{array}$ & $\begin{array}{l}\text { Vom Esel aus links ist der Hirsch. } \\
\text { As from the donkey left is the deer. }\end{array}$ & Subject \\
\hline & & $\begin{array}{l}\text { Vom Hirsch aus rechts ist der Esel. } \\
\text { As from the deer right is the donkey. }\end{array}$ & Object \\
\hline \multirow[t]{2}{*}{ AOS } & $\begin{array}{l}\text { Rechts von der Schlange aus ist der Hirsch. } \\
\text { Right as from the snake is the deer. }\end{array}$ & $\begin{array}{l}\text { Links vom Esel aus ist der Hirsch. } \\
\text { Left as from the donkey is the deer. }\end{array}$ & Subject \\
\hline & & $\begin{array}{l}\text { Rechts vom Hirsch aus ist der Esel. } \\
\text { Right as from the deer is the donkey. }\end{array}$ & Object \\
\hline
\end{tabular}


participants judged whether the layout conformed to the description (valid layout) or not (invalid layout). Then, a display announced that pressing the space bar would start the next trial.

Each item in its eight variants was assigned to a constant condition of the picture verification task (validity $\times$ presentation sequence). For one-dimensional layouts, valid and invalid $A-C$ and $C-A$ sequences were balanced across the conditions of the experimental design (anaphor $\times$ word order), as well as antecedent. Two-dimensional layouts were intended to be treated in the same way. Due to a coding mistake, valid and invalid layouts were correctly counterbalanced only in the $A-C$ condition, whereas all of the two-dimensional pictures in the $C-A$ condition were invalid ( 32 items), half of them being wrong on both dimensions and half of them being wrong on either the horizontal or the vertical dimension. As a consequence, the participants judged 48 valid layouts and 80 invalid layouts. It is unlikely, however, that this imbalance would induce an unwarranted systematic influence on P2 comprehension times, since it occurred after the critical P2 display. Furthermore, the imbalance in validity was equally distributed across all of the eight experimental conditions and should, if effective at all, affect all of the conditions alike.

\section{RESULTS}

\section{P2 Comprehension Times}

The analysis is based on the natural logarithm of P2 comprehension times for correct performance in the picture verification task. This was the case in $86 \%$ of all trials, ranging from $98 \%$ to $64 \%$ per participant. For each participant, latencies three standard deviations above the mean were classified as outliers and discarded from the analysis (less than $0.4 \%$ altogether). We will report on both the subject analysis $\left(F_{1}\right)$ and the item analysis $\left(F_{2}\right)$. Due to a missing value after aggregating data over participants, one item was excluded from the item analysis. Table 2 presents mean values of the subject analysis for untransformed data in milliseconds, together with log-transformed data. Untransformed data are plotted in Figure 1.

As predicted by relatum $=$ given, there was a main effect of anaphor in both the subject analysis $\left[F_{1}(1,24)=273.59\right.$, $\left.p<.001, \eta^{2}=.919\right]$ and the item analysis $\left[F_{2}(1,126)=\right.$ $\left.158.82, p<.001, \eta^{2}=.558\right]$. P2s with object anaphors were read faster than P2s with subject anaphors (5,854 vs. $7,415 \mathrm{msec})$. Likewise, the interaction of anaphor $\times$ word order was significant in the subject analysis $\left[F_{1}(3,72)=\right.$ $\left.26.77, p<.001, \eta^{2}=.527\right]$, as well as in the item analysis $\left[F_{2}(3,378)=18.36, p<.001, \eta^{2}=.127\right]$ (see Figure 1$)$. As predicted by given-new, this interaction was confirmed for the comparison of SOA with OSA $\left[F_{1}(1,24)=9.95\right.$, $p=.004, \eta^{2}=.293$, and $F_{2}(1,126)=5.311, p=.023$, $\left.\eta^{2}=.040\right]$. Although the adverb was in the sentence-final position in both conditions, $\mathrm{P} 2 \mathrm{~s}$ were read faster when the object anaphor was in the preverbal position (OSA), preceding the subject (given-new order), rather than in the postverbal position (SOA), following the subject (newgiven order). As predicted by Poset, the interaction of anaphor $X$ word order was also confirmed for the comparison of OSA with OAS $\left[F_{1}(1,24)=11.71, p=.002\right.$, $\eta^{2}=.328$, and $\left.F_{2}(1,126)=7.74, p=.006, \eta^{2}=.058\right]$. With a preverbal object anaphor, P2s were read faster if the adverb came earlier in the sentence (OAS) rather than late (OSA). Unexpectedly, the interaction of anaphor $\times$ word order was confirmed even for the comparison of OAS with AOS in the subject analysis $\left[F_{1}(1,24)=4.52\right.$, $\left.p=.044, \eta^{2}=.158\right]$, but it failed to reach significance in the item analysis $\left[F_{2}(1,126)=3.60, p=.060, \eta^{2}=.028\right]$. As is evident from Figure 1, however, this weakest and unpredicted effect has its source in the subject anaphor condition (nonlicensing context) and not in the object anaphor condition (licensing context). This is confirmed by pairwise comparisons of word order variants within the licensing context condition: SOA versus OSA $\left[t_{1}(24)=\right.$ $4.53, p<.001$, and $\left.t_{2}(126)=4.28, p<.001\right]$, OSA versus OAS $\left[t_{1}(24)=4.22, p<.001\right.$, and $t_{2}(126)=3.81$, $p<.001]$, and OAS versus AOS $\left(t_{1}\right.$ and $\left.t_{2}<1\right)$.

The analysis revealed one more effect - namely, a main effect of word order $\left[F_{1}(3,72)=6.80, p<.001, \eta^{2}=\right.$ .221 , and $\left.F_{2}(3,378)=8.48, p<.001, \eta^{2}=.063\right]$ (see Table 2). On average, P2s with SOA word order took longer to read than P2s with OSA word order $\left[F_{1}(1,24)=\right.$ $10.99, p=.003, \eta^{2}=.314$, and $F_{2}(1,126)=9.89, p=$ $.002, \eta^{2}=.073$ ], and P2s with AOS word order took longer to read than P2s with OAS word order $\left[F_{1}(1,24)=\right.$ $5.65, p=.026, \eta^{2}=.190$, and $F_{2}(1,126)=7.26, p=$ $\left..008, \eta^{2}=.054\right]$. OSA word order did not differ from OAS word order $\left[F_{1}(1,24)=2.56, p>.10\right.$, and $F_{2}(1,126)=$ $3.86, p=.052]$.

\section{P1 Comprehension Times}

In order to assess the pure word order effect, P1 comprehension times were analyzed. These were treated in the same way as were P2 comprehension times. P1 comprehension times were affected by word order (see Figure 1) $\left[F_{1}(3,72)=6.17, p=.001, \eta^{2}=.205\right.$, and $F_{2}(3,381)=$ $\left.5.01, p=.002, \eta^{2}=.038\right]$. Repeated contrasts revealed that P1s took longer to read with SOA than with OSA

Table 2

Mean P2 Comprehension Times of the Subject Analysis for Anaphor $\times$ Word Order (in Milliseconds) and Standard Deviations, Together With LogTransformed Data (in Italics)

\begin{tabular}{|c|c|c|c|c|c|c|c|c|}
\hline \multirow[b]{3}{*}{ Anaphor } & \multicolumn{8}{|c|}{ Word Order } \\
\hline & \multicolumn{2}{|c|}{ SOA } & \multicolumn{2}{|c|}{ OSA } & \multicolumn{2}{|c|}{ OAS } & \multicolumn{2}{|c|}{ AOS } \\
\hline & $M$ & $S D$ & $M$ & $S D$ & $M$ & $S D$ & $M$ & $S D$ \\
\hline \multirow[t]{2}{*}{ Subject } & 7,197 & 1,821 & 6,982 & 1,672 & 7,256 & 1,999 & 8,206 & 2,313 \\
\hline & 8.755 & .259 & 8.740 & .237 & 8.767 & .324 & 8.886 & .270 \\
\hline \multirow[t]{2}{*}{ Object } & 6,933 & 2,093 & 5,862 & 1,471 & 5,283 & 1,373 & 5,402 & 1,489 \\
\hline & 8.703 & .308 & 8.573 & .255 & 8.456 & .265 & 8.471 & .271 \\
\hline
\end{tabular}




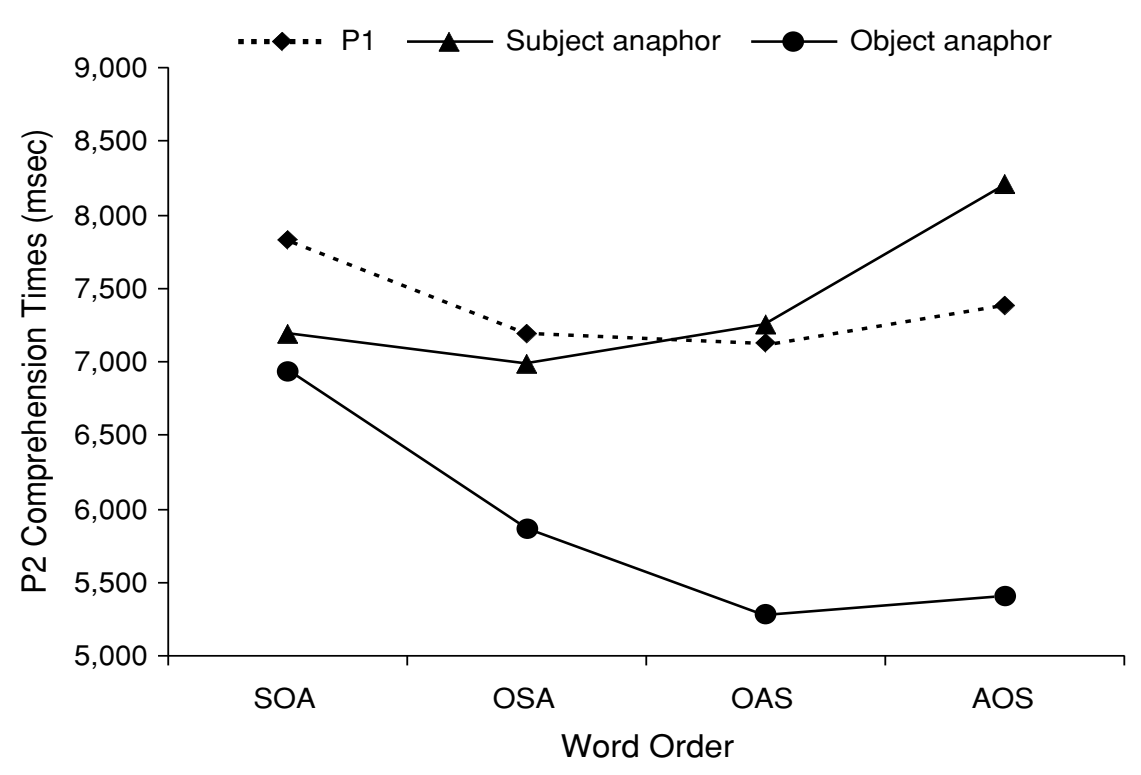

Figure 1. Mean untransformed comprehension times for P1, P2 with subject anaphor $($ relatum $=$ new), and $P 2$ with object anaphor $($ relatum $=$ given $)$, as a function of word order (SOA, OSA, OAS, and AOS). S, subject; O, object; A, adverb.

word order $(7,825$ vs. $7,193 \mathrm{msec})\left[F_{1}(1,24)=12.70, p=\right.$ $.002, \eta^{2}=.346$, and $F_{2}(1,127)=7.80, p=.006, \eta^{2}=$ $.058]$. The other two pairwise comparisons, OSA versus OAS and OAS versus AOS, were not significant.

\section{Accuracy}

For each participant (subject analysis) or item (item analysis), error rates were computed for each of the eight conditions of anaphor $\times$ word order and subjected to an ANOVA. There was a significant effect of anaphor in both the subject analysis $\left[F_{1}(1,24)=19.12, p<.001, \eta^{2}=.443\right]$ and the item analysis $\left[F_{2}(1,127)=9.747, p=.002, \eta^{2}=\right.$ $.071]$. P2s with an object anaphor (given relatum; 11.8\%) led to fewer errors than did P2s with a subject anaphor (new relatum; 16.2\%). The anaphor main effect was also reliable in both analyses if restricted to one-dimensional layouts, for which validity was correctly counterbalanced. No further effects emerged in these analyses.

\section{DISCUSSION}

One of the goals of our study was to further support the relatum $=$ given principle of premise integration (Hörnig et al., 2005) and to extend it to premises with spatial adverbs accompanied by an overt relatum expression. Our findings corroborated that a second premise is easier to comprehend if the involved relatum is given (object anaphor) rather than new (subject anaphor). We explain this result in terms of linguistic localization. The second premise of a pair tells the reasoner how to add the new entity relative to one of the two entities already given within the initial model. On this view, spatial premises are basically understood as placement instructions. This view is supported by a corresponding advantage of given relata with verbal placement instructions given to children (Huttenlocher \& Strauss, 1968) and to university students (Clark, 1972). In these studies, participants were faster and more often correct in adding a new entity to a given one if the latter was denoted by the relatum expression of the instruction rather than by the referent expression. Consonant with these findings, as well as with those reported by Hörnig et al. (2005), a given relatum not only sped up premise integration in comparison with a new relatum, but also led to more reliable models, indicated by fewer errors in picture verification.

The central question of the present study concerned the licensing conditions for noncanonical word order of spatial relational statements. Specifically, we were interested in whether noncanonical word order is licensed by given-new or by Poset. According to given-new, noncanonical word order meets information-structural demands by establishing a given-new order in the sentence. On this account, the contextual givenness of the entity denoted by the preverbal relatum expression is licensing noncanonical word order by virtue of the relatum expression's preceding the referent expression denoting the new entity, thereby yielding a given-new order. This explanation does not address the position of the spatial relational information conveyed by the adverb or the preposition. As a licensing device, given-new predicts that the given-new advantage in comprehension is larger for noncanonical word order, OSA, than for canonical word order, SOA, although the adverb is sentence final in both cases. Our results confirmed this prediction. Poset licensing cannot account for this difference. According to Poset, noncanonical word order is licensed if the preverbal constituent denotes a new entity that is Poset related to a given entity. In order to meet this condition, the relatum expression has to share 
the preverbal position with the adverb. Accordingly, contextual licensing of noncanonical OSA word order has to be attributed to given-new rather than Poset.

On the other hand, given-new licenses a preverbal relatum expression denoting the given entity regardless of whether the spatial relational expression is sentence final (OSA) or preverbal (OAS). In contrast, OAS is licensed by Poset, whereas OSA is not, since the preverbal constituent denotes a new place with OAS but not with OSA. Hence, Poset predicts that the licensing context improves comprehension of noncanonical OAS word order to a larger extent than it improves comprehension of noncanonical OSA word order, which is not Poset licensed. Our results confirmed this prediction too. This finding, in turn, cannot be explained by given-new, and we attribute it to Poset.

We conclude that both given-new and Poset licensed noncanonical word order, though not to the same extent. Poset licensing of noncanonical OAS (and AOS) word order was reliably stronger than given-new licensing of noncanonical OSA word order. Contextual licensing, therefore, is a matter of degree.

This explanation is consistent with our processing assumptions outlined above. Integrating the new referent of the second premise into the initial mental model starts by retrieving the given place occupied by the given relatum. Then a new place is added to the model, specified by the spatial relational expression. Finally, the new referent is put at the new place. Clark and Haviland (1977) explain the given-new advantage in terms of accelerated access to the given information in memory when it is encoded early in the sentence. From a processing perspective, accelerated access to the given relatum and to the place occupied by it ameliorates integration for both kinds of licensing, given-new and Poset. In case of Poset licensing, the early encoding of the spatial relational expression, in addition, speeds up the prefinal step of adding the new place to the model. Therefore, we recognize a qualitative difference between the processing of second premises with OSA word order (given-new licensing) and the processing of OAS or AOS word order (Poset licensing). At the same time, the two kinds of licensing are not independent of each other. Rather, the source of given-new licensing also contributes to Poset licensing. Like given-new licensing, Poset licensing profits from the earlier access to the given entity, but also from earlier initiation of relational processing - of adding the new place. This explains why Poset licensing is stronger than given-new licensing.

P2 comprehension times with spatial adverbs in the present experiment were considerably longer than ordinary reading times. P2 comprehension times with spatial prepositions in the experiment of Hörnig et al. (2005) were nearly as long. We see this as a reflection of the complexity of the integration process. In our opinion, these comprehension times do not compromise our interpretation that integration of a verbally presented second premise is affected by linguistic factors such as word order. On the contrary, despite the fact that comprehension implies complex processes, it proves to be highly sensitive to the influence of the linguistic form of the premise. It would nevertheless be worthwhile to ascertain the licensing conditions in different task environments.

Surprisingly, our data failed to support the assumption that spatial relational statements with a preverbal grammatical subject have canonical word order. Without prior context, SOA word order took longer to read than any other word order variant. This is even more surprising, given that Hörnig et al. (2005) obtained slightly though reliably shorter comprehension times for P1s with preverbal subjects than with preverbal objects $(5,384$ vs. $5,644 \mathrm{msec})$. It is not so easy, however, to assess unambiguously the difficulty of P1s with SOA word order. Canonical structures are usually much more frequent than noncanonical ones. In the present experiment, $75 \%$ of items had noncanonical word order (OSA, OAS, and AOS), whereas in Hörnig et al. this was the case for only half of the items. We suspect that the low frequency of premise pairs with "canonical" word order induced the apparent processing difficulty in the present experiment. This suspicion is nourished by the fact that in German, spatial relational statements are rarely expressed by means of an adverb supplemented by an overt relatum expression. Hence, it would be premature to reject the assumption that SOA word order is canonical. Since P2 was always parallel in structure to the preceding P1, P2 was less likely to be affected by frequency. This might explain why the assumed canonicity of SOA word order fits well with our licensing results, although it does not agree with P1 comprehension times. To control for word order effects, we regressed $\mathrm{P} 2$ comprehension times on $\mathrm{P} 1$ comprehension times $[R=.319 ; F(1,2691)=$ $301.295, p<.001]$ and reran the analyses on the residuals. These analyses confirmed the effects reported in the Results section.

The comparison of OAS with AOS yielded an unexpected interaction of word order $\times$ anaphor, indicating that the difference between the licensing and the nonlicensing conditions was larger for AOS than for OAS. This result would be compatible with the idea that contextual licensing is stronger for AOS than for OAS. The P2 comprehension time pattern, however, did not support this interpretation. Stronger contextual licensing bears on enhanced comprehensibility within the licensing condition, but OAS and AOS differed within the nonlicensing condition. P2s with a subject anaphor were less comprehensible with AOS word order than with OAS word order. Therefore, this result must be explained in terms of higher processing costs due to the inappropriate context. So far, we can offer no specific explanation. We note, however, that the increase in P2 comprehension times for unlicensed AOS word order, as opposed to SOA, corresponds to the increase for unlicensed noncanonical P2s with spatial prepositions in Hörnig et al. (2005), which most closely resembled our AOS word order.

The impaired comprehension of licensed OSA word order as opposed to licensed OAS word order might be due to a higher syntactic complexity of OSA word order. Specifically, the split prepositional phrase of OSA intro- 
duces a filler-gap dependency between the preverbal part of the prepositional phrase and the sentence-final part containing the adverb. Such an explanation, however, should apply regardless of whether the structures in question figure in an adequate context or not. In particular, the higher complexity of OSA than of OAS should have been expected to emerge as a word order main effect in P2 comprehension times, and should have led to longer P1 comprehension times as well. Premises with OSA word order, however, were not in general more difficult to comprehend than premises with OAS word order. Rather, impaired comprehension of OSA word order as opposed to OAS was limited to the licensing context condition.

On the basis of our study, we cannot decide whether our licensing results generalize to ordinary sentences with, for example, preverbal direct objects. The study was not intended to directly evaluate the Poset licensing originally proposed by Prince (1999). What is currently known is that given-new licensing of preverbal direct objects in German is comparatively weak (see Weskott, 2003). Possibly, Poset licensing of preverbal direct objects (e.g., in German) is stronger. ${ }^{4}$

Finally, we return to the question of how to account for the contextual licensing of a noncanonical second premise with a spatial preposition - namely the good comprehensibility of a second premise with a relatum expression denoting a given entity together with a preverbal spatial preposition, as in To the right of the deer $r_{\text {given }}$ is the don$k e y_{\text {new }}$ (Hörnig et al., 2005). As we have shown here, contextual licensing of a noncanonical second premise is stronger when the preverbal position contains both the relatum expression and the spatial relational expression (here, the spatial adverb) than when the preverbal position contains only the relatum expression. We have established a qualitative difference between these two conditions, Poset licensing versus given-new licensing. Only the former case, when the relatum expression shares the preverbal position with the spatial relational expression (adverb or preposition), identifies a new entity (i.e., a new place) as the denotatum of the preverbal constituent as a whole, the prepositional phrase. This early availability of the new place, rather than the early availability of merely the given relatum, substantially facilitates comprehension of a noncanonical second premise. This substantial facilitation is explained in terms of Poset licensing.

\section{REFERENCES}

Bader, M., Meng, M., Bayer, J., \& Hopf, J.-M. (2000). Syntaktische Funktionsambiguitäten im Deutschen: Ein Überblick. Zeitschrift für Sprachwissenschaft, 19, 34-102.

Clark, H. H. (1972). Difficulties people have in answering the question "Where is it?" Journal of Verbal Learning \& Verbal Behavior, 13, 133-137.

Clark, H. H., \& Haviland, S. E. (1977). Comprehension and the givennew contract. In R. O. Freedle (Ed.), Discourse processes: Advances in research and theory. Vol. 1: Discourse production and comprehension (pp. 1-40). Norwood, NJ: Ablex.

De VinzenCI, M. (1991). Syntactic parsing strategies in Italian. Dordrecht: Kluwer.
FraZIER, L. (1987). Syntactic processing: Evidence from Dutch. Natural Language \& Linguistic Theory, 5, 519-560.

GrÜNeISL, G., \& Zacharias, W. (Eds.) (1981). PA-Schnippelbuch Nr. 1. Nürnberg: Pädagogische Aktion.

Hemforth, B. (1993). Kognitives Parsing: Repräsentation und Verarbeitung sprachlichen Wissens. Sankt Augustin, Germany: Infix.

HersKovits, A. (1986). Language and spatial cognition: An interdisciplinary study of the prepositions in English. Cambridge, MA: Cambridge University Press.

Hörnig, R., Oberauer, K., \& Weidenfeld, A. (2005). Two principles of premise integration in spatial reasoning. Memory \& Cognition, 33, 131-139.

Huttenlocher, J., \& Higgins, E. T. (1971). Adjectives, comparatives, and syllogisms. Psychological Review, 78, 487-504.

Huttenlocher, J., \& Strauss, S. (1968). Comprehension and a statement's relation to the situation it describes. Journal of Verbal Learning \& Verbal Behavior, 7, 527-530.

HyöNÄ, J., \& HuJANEN, H. (1997). Effects of case marking and word order on sentence parsing in Finnish: An eye fixation analysis. Quarterly Journal of Experimental Psychology, 50A, 841-858.

Johnson-LaIRD, P. N. (1983). Mental models: Towards a cognitive science of language, inference, and consciousness. Cambridge, MA: Harvard University Press.

Miller, G. A., \& Johnson-Laird, P. N. (1976). Language and perception. Cambridge: Cambridge University Press.

Prince, E. F. (1998). On the limits of syntax, with reference to leftdislocation and topicalizaton. In P. Culicover \& L. McNally (Eds.), Syntax and semantics: Vol. 29. The limits of syntax (pp. 281-302). New York: Academic Press.

Prince, E. F. (1999). How not to mark topics: "Topicalization" in English and Yiddish. In Texas Linguistic Forum. Austin: University of Texas. Available at www.ling.upenn.edu/ ellen/home.html.

SCHILDER, F., \& TenbrinK, T. (2002, November). The interplay of information structure and the placement of after and before. Paper presented at the Workshop on Information Structure in Context, Stuttgart. Stuttgart: University of Stuttgart. Available at www.informatik.uni-bremen. de/ tenbrink/.

Schlesewsky, M., Fanselow, G., Kliegl, R., \& Krems, J. (2000). The subject preference in the processing of locally ambiguous whquestions in German. In B. Hemforth \& L. Koniecny (Eds.), German sentence processing (pp. 65-93). Dordrecht: Kluwer.

WeSKotT, T. (2003). Information structure as a processing guide. $\mathrm{PhD}$ thesis, University of Leipzig.

Yekovich, F., R., Walker, C. H., \& Blackman, H. S. (1979). The role of presupposed and focal information. Journal of Verbal Learning \& Verbal Behavior, 18, 535-548.

\section{NOTES}

1. The obvious candidate for a given-new "licensing" context for canonical word order SOA would be the subject anaphor condition, since the canonical sentence has given-new order in this condition. Indeed, Yekovich, Walker, and Blackman (1979) have shown that ordinary sentences with canonical word order (e.g., The shark attacked the diver near the reef) are easier to comprehend with given-new order (subject anaphor) than with new-given order (object anaphor). For the statistical analysis, however, it is of no concern which one of the anaphor conditions is "licensing" SOA word order and which one is not. The anaphor $\times$ word order interaction will attest to whether the difference between the "licensing" and the nonlicensing condition is larger for one of the two word order conditions.

2. There was neither a main effect of antecedent nor any interaction in the overall analysis. There are reasons to assume that antecedent plays a role at least for descriptions of linear layouts. In order for these descriptions to be determinate, the spatial adverbs in the two premises must express the same relation whenever the antecedent and anaphor are of different grammatical function, but they must express converse relations whenever the antecedent and anaphor are of the same grammatical function. We therefore conducted a separate analysis for descriptions of linear layouts only. In this analysis as well, there was no effect at all of antecedent. 
3. We used the stimulus material of Hörnig et al. (2005). The pictures used in the verification task were taken from a commercial sampler of black and white drawings (Grüneisl \& Zacharias, 1981). These drawings had been scanned and transformed to a $120 \times 120$ pixel PCX format.

4. For English sentences, Schilder and Tenbrink (2002) examined the contextual conditions of placing temporal relational adverbs (after and before) in the sentence-initial position. These authors concluded that the sentence-initial occurrences of temporal adverbs are Poset licensed. This is interesting, insofar as temporal adverbs, on the one hand, contribute to the structuring of events in discourse and therefore meet the Poset conditions advocated by Prince more closely than the spatial adverbs examined here. On the other hand, it is a small step from conceiving of temporal relations as Poset relations to accepting spatial relations as a proper instance of Poset relations too.

(Manuscript received July 21, 2004;

revision accepted for publication June 10,2005 .) 